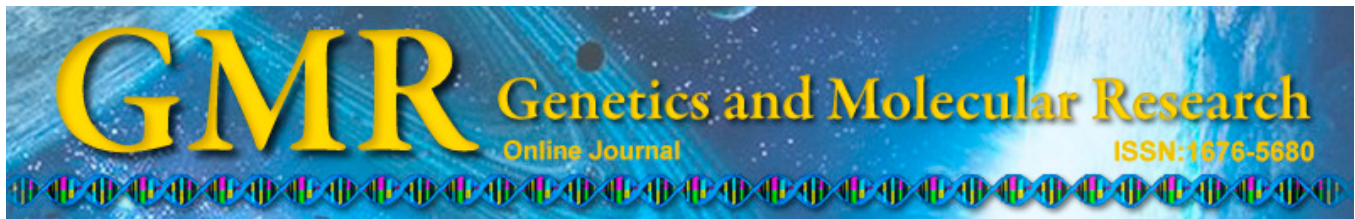

\title{
Identification of microRNAs as diagnostic biomarkers in screening of head and neck cancer: a meta-analysis
}

\author{
M. Zhang ${ }^{1}$, L.J. Zhao ${ }^{1}$, W.Q. Liang ${ }^{2}$ and Z.P. Mao ${ }^{1}$ \\ ${ }^{1}$ Department of Otolaryngology-Head and Neck Surgery, \\ Shaoxing Second Hospital, Shaoxing, China \\ ${ }^{2}$ Department of Orthopaedics, Shaoxing People's Hospital, \\ Shaoxing Hospital of Zhejiang University, Shaoxing, Zhejiang Province, China \\ Corresponding author: Z.P. Mao \\ E-mail: mzp_ssh@163.com
}

Genet. Mol. Res. 14 (4): 16562-16576 (2015)

Received January 12, 2015

Accepted June 17, 2015

Published December 11, 2015

DOI http://dx.doi.org/10.4238/2015.December.11.3

\begin{abstract}
Head and neck cancer (HNC) is one of the most prevalent cancers; it is often diagnosed at its advanced stage and has a low 5-year survival rate. Evidence suggests that noninvasive biomarker microRNAs (miRNAs) are valuable for early diagnosis of HNC. This meta-analysis assessed the diagnostic value of miRNAs in $\mathrm{HNC}$ detection. A systematic literature search for relevant studies up to August 4, 2014 was conducted in databases and other sources. Statistical analysis was conducted using STATA 12.0. Pooled sensitivity, specificity, and other parameters, together with a summary receiver operating characteristic curve were used to assess the overall performance of miRNA assays. Subgroup analyses and meta-regression were used to analyze heterogeneity, and a Deeks' funnel plot asymmetry test assessed publication bias. Twenty-four articles with $1856 \mathrm{HNC}$ patients and 1375 controls were included. The pooled results were as follows: sensitivity, $0.80(95 \% \mathrm{CI}$ $=0.77-0.83)$; specificity, $0.80(95 \% \mathrm{CI}=0.76-0.85)$; positive likelihood ratio, $4.1(95 \% \mathrm{CI}=3.2-5.2)$; negative likelihood ratio, $0.25(95 \% \mathrm{CI}$
\end{abstract}


$=0.21-0.30)$; diagnostic odds ratio (DOR), $16(95 \% \mathrm{CI}=11-24)$; and area under curve (AUC), $0.87(95 \% \mathrm{CI}=0.84-0.89)$. We conducted subgroup analyses based on ethnicity, cancer type, miRNA profiling, and specimen types, and found that miRNA assays yielded the highest accuracy in esophageal cancer. Notably, the DOR was 99 and the AUC was 0.96 for the multiple miRNA test, indicating strong discrimination of cancer patients from healthy people. The meta-analysis indicates that noninvasive miRNAs are a promising diagnostic tool with moderate accuracy for HNC diagnosis.

Key words: MicroRNAs; Head and neck cancer; Screening;

Meta-analysis

\section{INTRODUCTION}

Head and neck cancer (HNC), which includes esophageal, oral, thyroid, laryngeal, nasopharyngeal, and parotid cancers, as well as retinoblastoma, is estimated to be the sixth most common malignant tumor, as well as the sixth leading cause of cancer-related deaths in the world (Parkin, 2001; Jemal et al., 2008). There are approximately 482,300 new esophageal cancer cases and 406,800 esophageal cancer-related deaths every year, and the annual incidence of oral cancer is estimated at more than 500,000. The etiology of HNC is not fully understood but major contributory risk factors are thought to be tobacco smoking, excess alcohol consumption, and betel quid usage. The risk of HNC is 38 times higher in heavy smokers and drinkers than in people who neither smoke nor drink. The 5-year survival rate for HNC has remained around $50 \%$ over the past decades, despite advances in cancer diagnosis and treatment (Hardisson, 2003). Furthermore, the 5-year survival rate for early-stage esophageal cancer patients is more than $90 \%$, but drops to $15 \%$ when the cancer has developed into the advanced stage. This implies that accurate early diagnosis is crucial to improving the survival rate of patients, thereby highlighting the need to find powerful biomarkers for early detection.

In recent years, a great deal of effort has been made to identify potential diagnostic biomarkers for HNC by gene expression profiling technologies. However, the lack of sensitivity and specificity limits their usefulness in clinical examinations. At present, the primary screening tool for esophageal cancer is endoscopy, by which a subset of patients can be diagnosed at the early stages and can therefore receive timely treatment, which greatly improves their chances of survival. However, endoscopy is inconvenient because it may cause discomfort or even pain in the patient (Zhou and Wang, 2010). The current gold standard for thyroid cancer detection is fine needle aspiration biopsy. However, it is often impossible to determine malignancy with a high degree of certainty: only $70-80 \%$ of cases can be definitively determined. Therefore, novel and noninvasive biomarkers with high accuracy for the early diagnosis of HNC are urgently needed to reduce morbidity and mortality.

MicroRNAs (miRNAs), a class of mature, non-coding, single-strand RNAs with a typical length of 22 nucleotides, have opened up a new field for the molecular diagnosis of cancer (Kloosterman and Plasterk, 2006; Stefani and Slack, 2008). With the capacity to target hundreds of genes, they play an important role in a variety of biological processes, such as cell differentiation, proliferation, and apoptosis, and it is not surprising that many studies 
have now identified an association between dysregulation of miRNAs and carcinogenesis, including that of HNC (Miska, 2005; Du and Zamore, 2007; Avissar et al., 2009; Keutgen et al., 2012; Gombos et al., 2013; Hirajima et al., 2013). miRNAs are also expressed stably in plasma, serum, and other body fluids, indicating their potential use as clinical biomarkers.

Although many studies have identified the potential of miRNAs as biomarkers in $\mathrm{HNC}$ detection, study results are inconsistent owing to differences in study design, different specimen types, and different miRNAs. Indeed, different groups may also result in conflicting conclusions. Therefore, we conducted this meta-analysis to systematically evaluate the overall diagnostic accuracy of miRNAs in HNC detection.

\section{MATERIAL AND METHODS}

\section{Publication search}

We conducted a systematic literature search of PubMed, Wiley Online Library, EMBASE, Web of Science, and the China National Knowledge Infrastructure database up to August 4, 2014. The following key words were used: ("head and neck neoplasms" or "facial neoplasms" or "mouth neoplasms" or "parathyroid neoplasms" or "thyroid cancer" or "oral cancer" or "esophageal neoplasms") and ("microRNAs" or "miRNA" or "miRs" or "miR*") and ("sensitivity" or "specificity" or "diagnostic value" or "diagnosis" or "ROC curve"). Additional relevant studies from the reference lists were also included in our meta-analysis.

\section{Inclusion criteria}

Studies were required to meet the following inclusion criteria to be eligible for our meta-analysis: 1) they were human case-control studies; 2) they were clinical studies relating to the diagnostic value of miRNAs in HNC; 3 ) the studies made available information on true positives, false positives, false negatives, and true negatives; 4) publications were not duplications; and 5) studies were not in the form of reviews, abstracts, or editorial articles.

\section{Data extraction and quality assessment}

The necessary information and data were extracted from the final eligible articles as follows: first author, the year of publication, the country of origin, subjects' ethnicity, the number of cases and controls, cancer types, source of control, miRNA profiling, specimen, expression change, and data needed for diagnostic meta-analysis (sensitivity and specificity). The methodological qualities of the selected eligible articles were assessed by the Quality Assessment of Diagnostic Accuracy Studies 2 (QUADAS-2) score system. The QUADAS-2 tool combines the index of patient selection, the index test, the reference standard, and flow and timing to evaluate risk of bias and applicability concerns.

\section{Statistical analysis}

The statistical analyses in our study were performed using the STATA 12.0 statistical software. The pooled parameters sensitivity, specificity, positive likelihood ratio (PLR), negative likelihood ratio (NLR), and diagnostic odds ratio (DOR) and their 95\%CIs were 
calculated to evaluate the overall diagnostic accuracy, as well as the summary receiver operator characteristic curve and the area under the curve (AUC). Pairs of sensitivity and specificity were presented as forest plots to identify the heterogeneity between studies using a randomeffects approach. As the random error or differences in clinical or methodological characteristics of studies resulted in conflicting outcomes, subgroup analyses and meta-regression were conducted to explore the source of heterogeneity. Additionally, sensitivity analysis was performed to assess the robustness of our analysis and the publication bias of selected studies was assessed using the Deeks' funnel plot asymmetry test.

\section{RESULTS}

\section{Included studies}

The flow diagram of the selected studies is shown in Figure 1. A total of 649 articles were identified from databases and other sources, among which 104 duplicates were excluded. Of the remaining 545 articles, 466 were excluded, including 322 abstracts, reviews, and metaanalyses, and 144 irrelevant studies; and 79 studies were left for further full-text examination. After reading the texts carefully, 35 prognostic studies and 20 studies without sufficient data were removed. Finally, 24 eligible studies were included in our meta-analysis (Avissar et al., 2009; Komatsu et al., 2011; Keutgen et al., 2012; Kitano et al., 2011, 2012; Lu et al., 2012; Maclellan et al., 2012; Shen et al., 2012; Vriens et al., 2012; Zeng et al., 2012; Gombos et al., 2013; Hirajima et al., 2013; Mazeh et al., 2011; Ouyang et al., 2013; Sheinerman et al., 2013; Takeshita et al., 2013; Wang et al., 2013; Zhang et al., 2010, 2011, 2013; Liu et al., 2010, 2013, 2014; Ries et al., 2014).

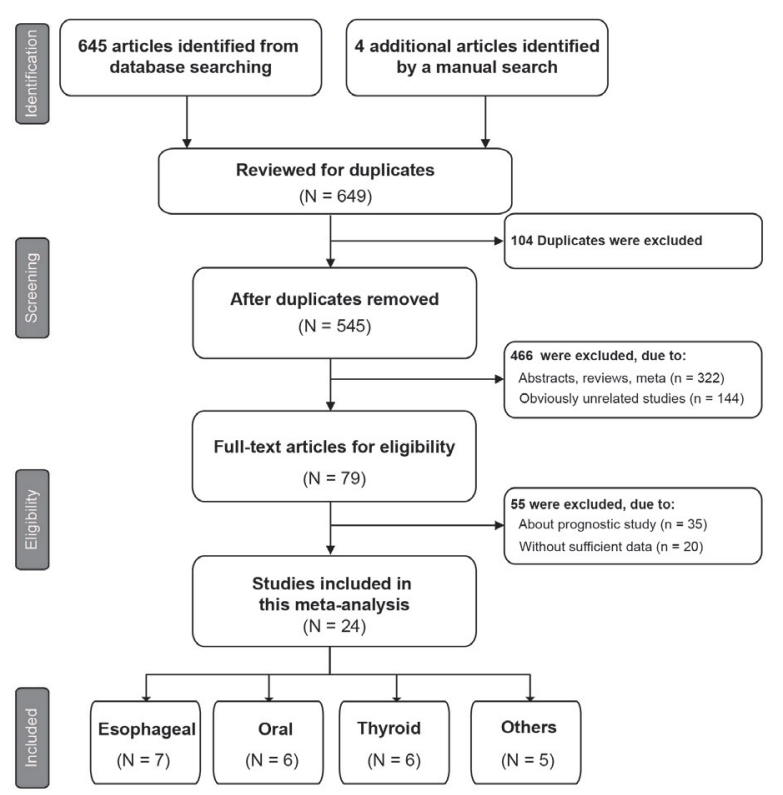

Figure 1. Flow chart of study selection. 


\section{Study characteristics and quality assessment}

Table 1 shows the characteristics of eligible studies published between 2009 and 2014. The 24 articles comprised 69 studies including 1856 patients with HNC and 1375 controls. Of the 24 diagnostic articles, different ethnic groups were involved: 13 focused on Asians and the remaining 11 articles recruited Caucasian participants. The expression levels of miRNA were analyzed by quantitative reverse transcription polymerase chain reaction (qRT-PCR) in all the articles studying esophageal cancer $(\mathrm{N}=7)$, thyroid cancer $(\mathrm{N}=6)$, oral cancer $(\mathrm{N}=6)$, and other cancers $(\mathrm{N}=5)$. Eight of the 24 articles measured single miRNA expression levels between cases and controls, while the remaining 16 investigated the diagnostic performance of multiple miRNAs. Among the 24 articles, plasma specimens were used to measure the miRNA expression level in eight studies, while serum specimens were used in six studies, and tissue specimens were used in the remaining 10 studies. The QUADAS-2 scores in Table 1 show that all studies had moderately high scores, indicating the relatively high quality of the studies included. Further, as the bar graph in Figure 2 reveals, risk of bias including index subject bias, test bias, reference bias, and diagnosis bias of each single study was also relatively low, and subject applicability, test applicability, and reference applicability in the applicability domain were high, suggesting the reliability of our studies included.

\section{Diagnostic accuracy of miRNAs in HNC}

Forest plots of sensitivity and specificity for miRNA assays in HNC detection were conducted, and the heterogeneity analysis revealed $I^{2}$ values of $83.46(95 \% \mathrm{CI}=80.04-86.88$; $\mathrm{P}<0.01)$ for sensitivity and $87.76(95 \% \mathrm{CI}=85.43 .84-90.08 ; \mathrm{P}<0.01)$ for specificity, which indicates significant heterogeneity. Therefore, the random-effect model was selected for this study. The summary assessments of miRNAs in the diagnosis of HNC are shown in Table 2. The pooled sensitivity was $0.80(95 \% \mathrm{CI}=0.77-0.83)$ and the specificity was $0.80(95 \% \mathrm{CI}=$ 0.76-0.85). The summary receiver operating characteristic curve was plotted with an AUC value of $0.87(95 \% \mathrm{CI}=0.84-0.89)$, indicating moderate accuracy for miRNAs assays in HNC detection. The PLR and NLR, which have been considered more clinically meaningful than sensitivity or specificity, were $4.1(95 \% \mathrm{CI}=3.2-5.2)$ and $0.25(95 \% \mathrm{CI}=0.21-0.30)$, respectively in our study, meaning that a person with $\mathrm{HNC}$ is four-times more likely to have a positive test result than a healthy person, while a healthy person is four-times more likely to have a negative test result than a person with HNC. The DOR, which is a combination of NLR and PLR, was $16(95 \% \mathrm{CI}=11-24)$, indicating a moderate level of diagnostic accuracy.

\section{Subgroup analyses}

The subgroup analyses shown in Table 2 were conducted for further comparison. Subgroup analysis according to ethnicity was performed first. The specificity for Asian subjects was 0.82 and the PLR was 4.5 , whereas for Caucasians those figures were 0.79 and 3.9, respectively, but there were minimal differences in the other parameters. These data indicated that the accuracy of miRNA assays for HNC in the Asian population was slightly better than in the Caucasian population.

Secondly, we investigated subgroup analysis according to cancer types for esophageal cancer, thyroid cancer, oral cancer, and other cancers. For the miRNA assays in esophageal 
miRNAs in screening of head and neck cancer

16567

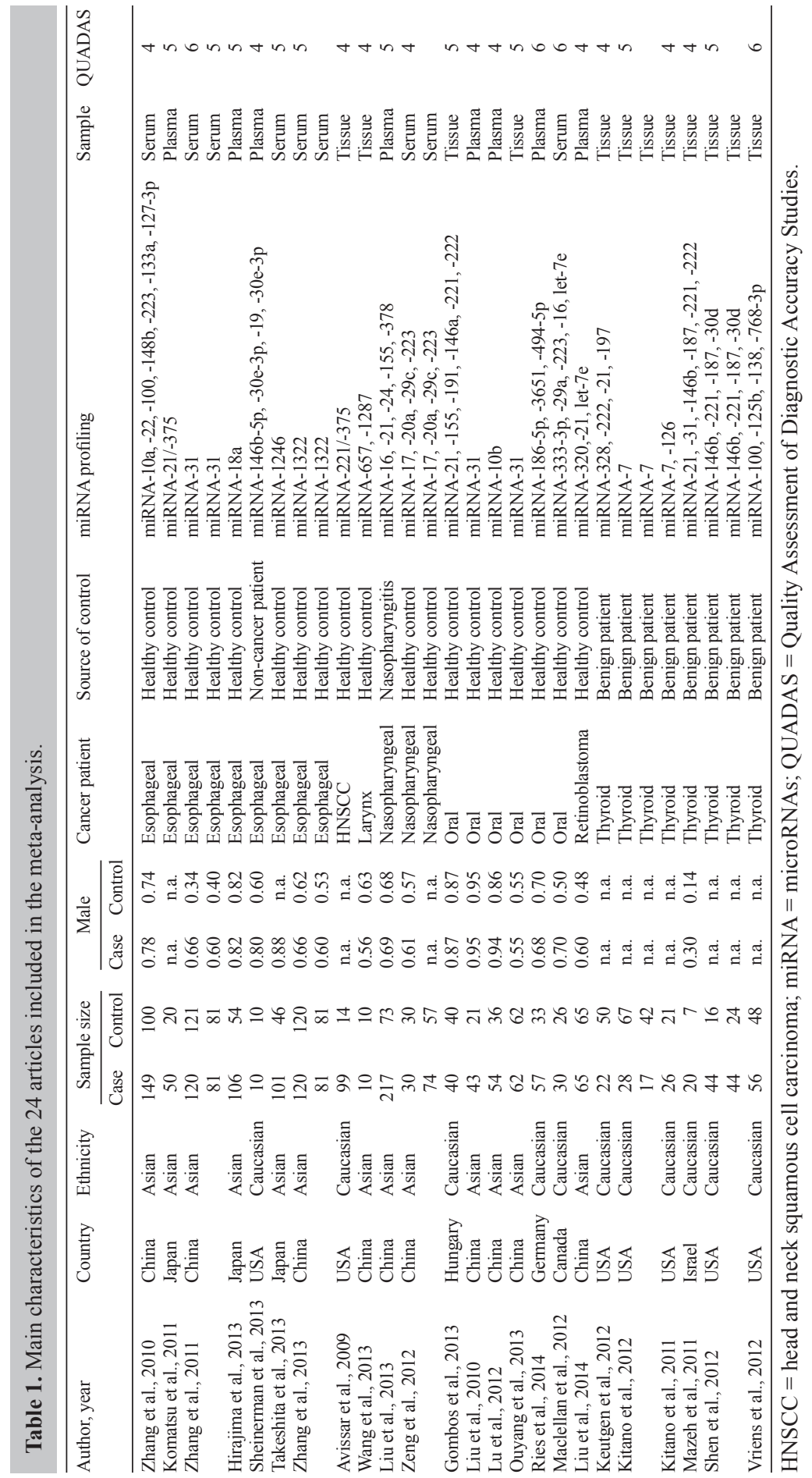


cancer, the pooled sensitivity, specificity, PLR, NLR, DOR, and AUC were $0.81(95 \% \mathrm{CI}=$ $0.76-0.84), 0.86(95 \% \mathrm{CI}=0.82-0.89), 5.6(95 \% \mathrm{CI}=4.3-7.2), 0.23(95 \% \mathrm{CI}=0.18-0.28), 25$ $(95 \% \mathrm{CI}=16-37)$, and $0.90(95 \% \mathrm{CI}=0.87-0.92)$, respectively (Figure $3 \mathrm{~A})$. For the miRNA assays in thyroid cancer, the corresponding pooled parameters were $0.80(95 \% \mathrm{CI}=0.72-0.86)$, $0.80(95 \% \mathrm{CI}=0.69-0.88), 4.0(95 \% \mathrm{CI}=2.6-6.2), 0.25(95 \% \mathrm{CI}=0.18-0.35), 16(95 \% \mathrm{CI}=$ $9-28)$, and $0.87(95 \% \mathrm{CI}=0.83-0.89)$, respectively (Figure 3B). For the miRNA assays in oral cancer, the corresponding pooled results were $0.78(95 \% \mathrm{CI}=0.71-0.84), 0.72(95 \% \mathrm{CI}=0.59$ $0.83), 2.8(95 \% \mathrm{CI}=1.8-4.5), 0.30(95 \% \mathrm{CI}=0.20-0.44), 9(95 \% \mathrm{CI}=4-21)$, and $0.82(95 \% \mathrm{CI}$ $=0.79-0.85$ ), respectively (Figure 3C). For the miRNA assays in other HNCs, the AUC was $0.88(0.85-0.91)$ (Figure 3D). We found that miRNAs assays in esophageal cancer yielded relatively higher diagnostic accuracy than any other HNC type.

\section{(A) Risk of Bias}

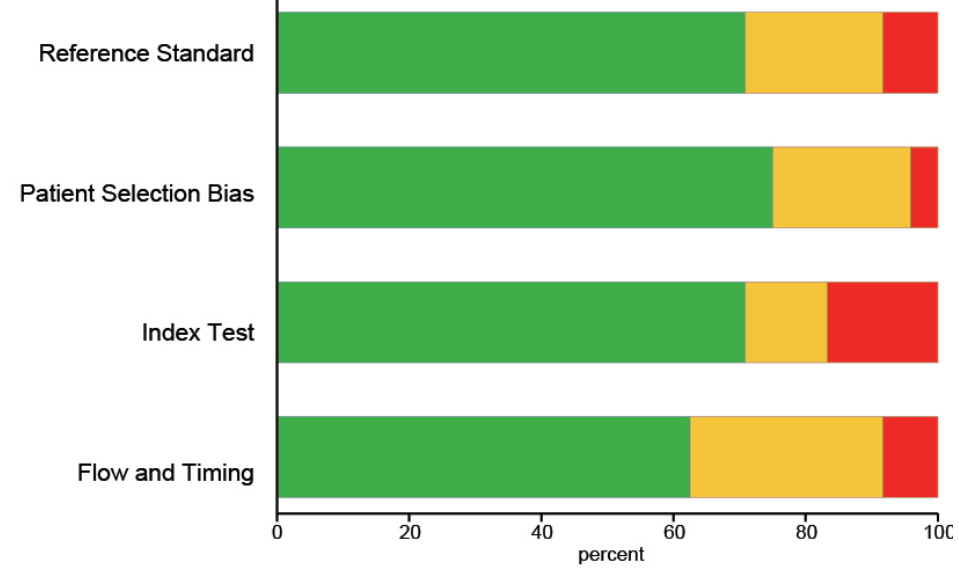

(B) Applicability

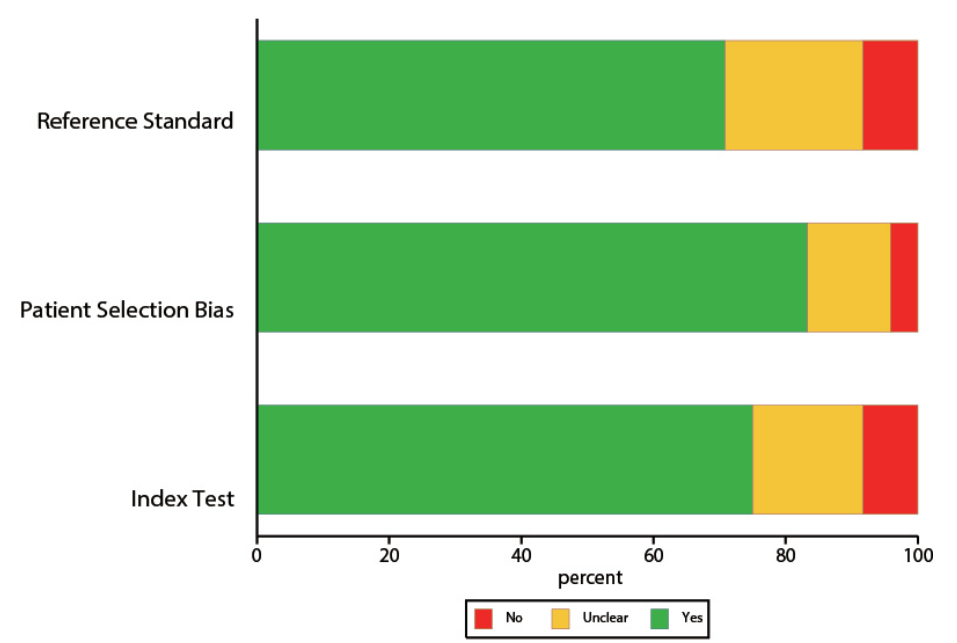

Figure 2. Bar graphs of the quality assessment of studies included using the Quality Assessment of Diagnostic Accuracy Studies 2 (QUADAS-2) score system (A. risk of bias; B. applicability). 
Table 2. Summary of estimates of diagnostic criteria and their $95 \%$ confidence intervals $(95 \% \mathrm{CIs})$.

\begin{tabular}{lcccccc}
\hline Analysis & SEN $(95 \% \mathrm{CI})$ & SPE $(95 \% \mathrm{CI})$ & PLR $(95 \% \mathrm{CI})$ & NLR $(95 \% \mathrm{CI})$ & DOR $(95 \% \mathrm{CI})$ & AUC $(95 \% \mathrm{CI})$ \\
\hline Ethnicity & & & & & \\
$\quad$ Asian & $0.80(0.76-0.84)$ & $0.82(0.77-0.87)$ & $4.5(3.3-6.1)$ & $0.24(0.19-0.31)$ & $19(11-31)$ & $0.88(0.85-0.90)$ \\
$\quad$ Caucasian & $0.80(0.75-0.84)$ & $0.79(0.71-0.86)$ & $3.9(2.6-5.6)$ & $0.25(0.19-0.33)$ & $15(9-27)$ & $0.86(0.83-0.89)$ \\
Cancer types & & & & & \\
$\quad$ Esophageal cancer & $0.81(0.76-0.84)$ & $0.86(0.82-0.89)$ & $5.6(4.3-7.2)$ & $0.23(0.18-0.28)$ & $25(16-37)$ & $0.90(0.87-0.92)$ \\
$\quad$ Thyroid cancer & $0.80(0.72-0.86)$ & $0.80(0.69-0.88)$ & $4.0(2.6-6.2)$ & $0.25(0.18-0.35)$ & $16(9-28)$ & $0.87(0.83-0.89)$ \\
$\quad$ Oral cancer & $0.78(0.71-0.84)$ & $0.72(0.59-0.83)$ & $2.8(1.8-4.5)$ & $0.30(0.20-0.44)$ & $9(4-21)$ & $0.82(0.79-0.85)$ \\
$\quad$ Other HNC & $0.83(0.73-0.90)$ & $0.80(0.67-0.89)$ & $4.1(2.2-7.7)$ & $0.22(0.12-0.39)$ & $19(6-62)$ & $0.88(0.85-0.91)$ \\
miRNA profiling & & & & & \\
$\quad$ Single miRNA & $0.77(0.73-0.80)$ & $0.76(0.71-0.81)$ & $3.3(2.6-4.1)$ & $0.30(0.26-0.36)$ & $11(7-16)$ & $0.83(0.79-0.86)$ \\
$\quad$ Multiple miRNA & $0.90(0.85-0.93)$ & $0.92(0.86-0.95)$ & $10.9(6.2-19.1)$ & $0.10(0.07-0.16)$ & $99(44-225)$ & $0.96(0.94-0.97)$ \\
Specimen types & & & & & \\
$\quad$ Plasma-based & $0.77(0.70-0.82)$ & $0.69(0.56-0.79)$ & $2.5(1.6-3.8)$ & $0.34(0.23-0.49)$ & $7(3-16)$ & $0.80(0.76-0.83)$ \\
$\quad$ Serum-based & $0.81(0.76-0.86)$ & $0.85(0.81-0.88)$ & $5.3(4.2-6.7)$ & $0.22(0.17-0.28)$ & $24(16-38)$ & $0.90(0.87-0.92)$ \\
$\quad$ Tissue-based & $0.81(0.75-0.86)$ & $0.82(0.74-0.87)$ & $4.4(3.1-6.3)$ & $0.23(0.17-0.31)$ & $19(11-33)$ & $0.88(0.85-0.91)$ \\
$\quad$ Pooled & $0.80(0.77-0.83)$ & $0.80(0.76-0.85)$ & $4.1(3.2-5.2)$ & $0.25(0.21-0.30)$ & $16(11-24)$ & $0.87(0.84-0.89)$ \\
$\quad$ Outliers excluded & $0.79(0.76-0.82)$ & $0.80(0.77-0.83)$ & $4.0(3.3-4.8)$ & $0.26(0.22-0.30)$ & $16(11-21)$ & $0.87(0.83-0.89)$ \\
\hline
\end{tabular}

$\mathrm{HNC}=$ head and neck cancer; $\mathrm{SEN}=$ sensitivity; $\mathrm{SPE}=$ specificity PLR $=$ positive likelihood ratio; NLR = negative likelihood ratio; $\mathrm{DOR}=$ diagnostic odds ratio; $\mathrm{AUC}=$ area under curve; miRNA $=$ microRNA.
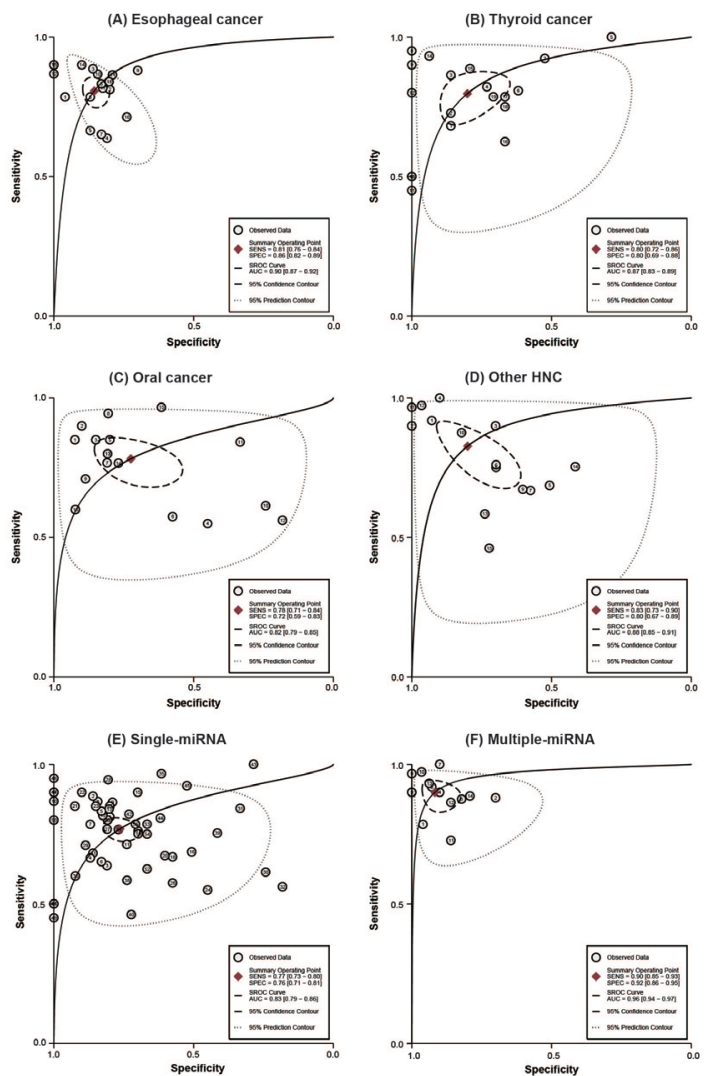

Figure 3. Symmetric receiver operating characteristic curve of microRNAs (miRNAs) describing the diagnostic performance [A. esophageal cancer; B. thyroid cancer; C. oral cancer; D. other head and neck cancer (HNC); E. single miRNA; and F. multiple miRNA]. 
We then took miRNA profiling into consideration in the subgroup analysis. The results indicated that the multiple-miRNA assay was significantly more accurate than the singlemiRNA assay: sensitivity increased from 0.77 to 0.90 , specificity increased from 0.76 to 0.92 , PLR increased from 3.3 to 10.9 , NLR decreased from 0.30 to 0.10 , DOR increased from 11 to 99 , and AUC increased from 0.83 to 0.96 (Figure $3 \mathrm{E}$ and $\mathrm{F}$ ). The encouraging results of the multiple-miRNA assays $($ AUC $=0.96)$ may provide solid evidence for the clinical value of miRNAs as biomarkers in early-stage HNC diagnosis, and the multiple-miRNA test for HNC diagnosis is worthy of future attention.

Finally, subgroup analysis was conducted on specimen types and we found that serum-based and tissue-based tests were more sensitive ( 0.81 vs 0.81 vs 0.77 , respectively) and specific ( 0.85 vs 0.82 vs 0.69 , respectively) than the plasma-based test, suggesting that serum and tissue may be better matrices for HNC detection.

Further, as different study designs were chosen in different laboratories, which may have been a source of heterogeneity, meta-regression analysis was used to identify the sources of heterogeneity. As shown in Figure 4, the results suggested ethnic origin $(\mathrm{P}<0.001)$, cancer type $(\mathrm{P}<0.001)$, miRNA profiling $(\mathrm{P}<0.001)$, and specimen type $(\mathrm{P}<0.001)$ were the sources of heterogeneity in sensitivity, as well as in specificity, which also confirmed the subgroup analyses in our study.
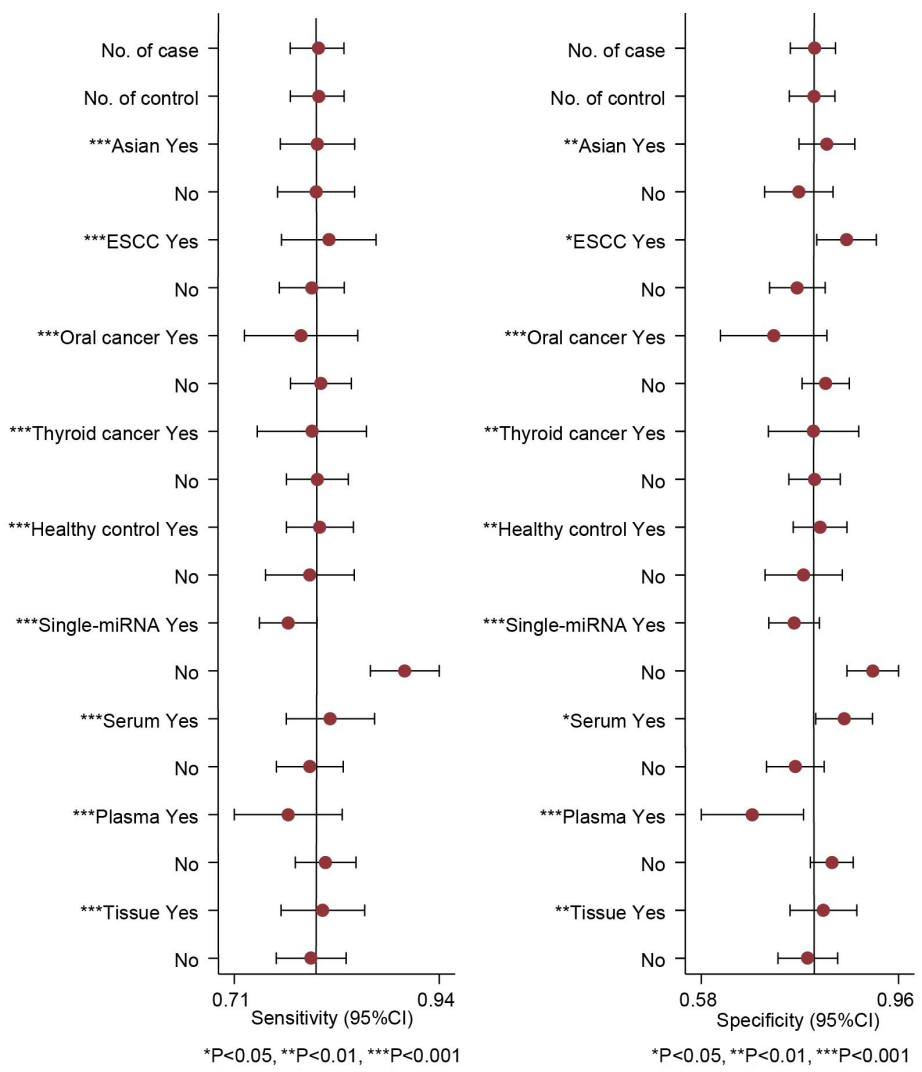

Figure 4. Meta-regression to explore the heterogeneity between studies. 


\section{Sensitivity analysis and publication bias}

Sensitivity analysis was performed to assess the stability of our study. The data shown in Figure 5 suggest that the 10 studies included might have influenced the pooled results. When we deleted those 10 outlines from the analysis to assess the influence of the removed data set on the overall result, the diagnostic parameters remained almost the same as before (Table 2), which confirmed the robustness of our analysis. As shown in Figure 6, with a pretest probability of $25 \%$, the post-test probability of HNC using multiple miRNAs for a positive test result was $76 \%$, while the probability of a negative test result was $4 \%$; the post-test probabilities were moderate, indicating that the multiple miRNA assay can serve as a good indicator in HNC detection. The funnel plots in Figure 7 were calculated to access the publication bias of the studies. The slope coefficient was associated with a $\mathrm{P}$ value of 0.24 , which suggested no publication bias in our study.

(a) Goodness of Fit

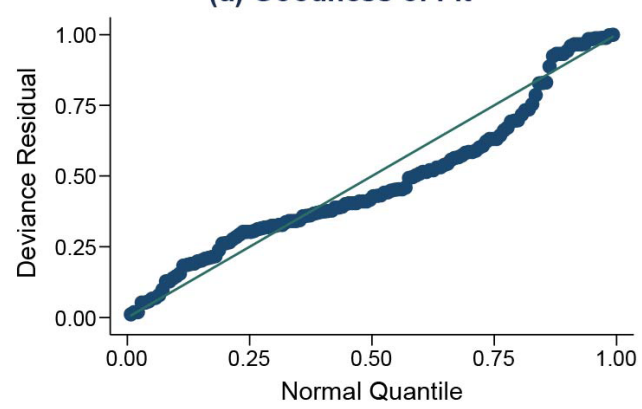

(c) Influence Analysis

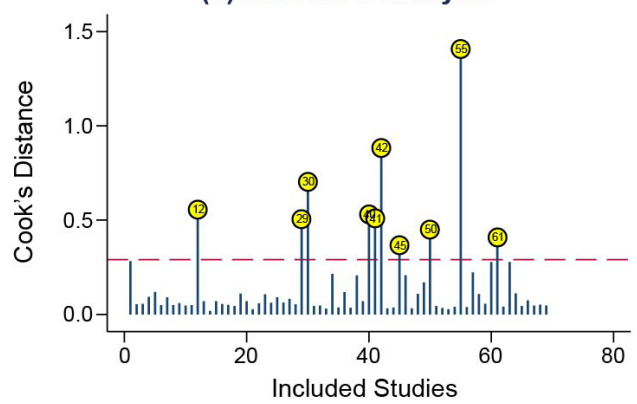

(b) Bivariate Normality

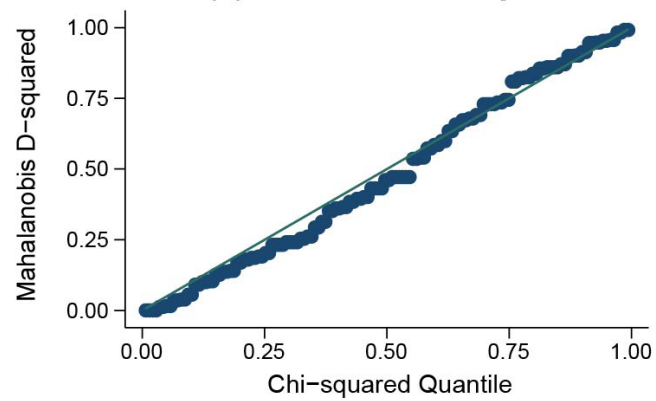

(d) Outlier Detection

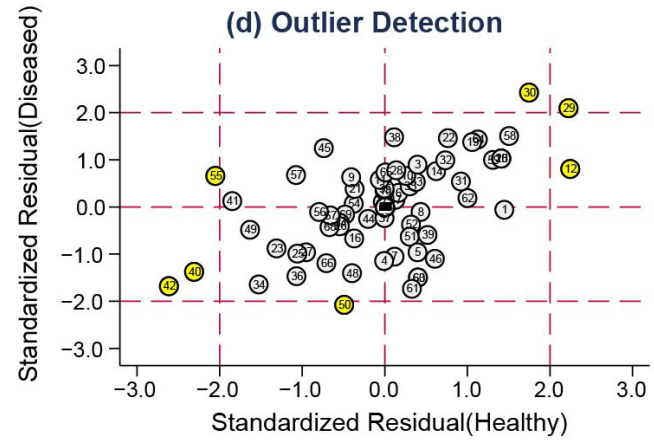

Figure 5. Sensitivity analysis goodness-of-fit (a); bivariate normality (b); influence analysis (c); outlier detection (d).

\section{DISCUSSION}

$\mathrm{HNC}$ is one of the most common cancers worldwide with a high incidence and mortality. The 5-year survival rate for patients with advanced tumors is less than $15 \%$ : much lower than patients diagnosed with early-stage $\mathrm{HNC}$ with its $90 \%$ survival rate. However, to the best 
M. Zhang et al.

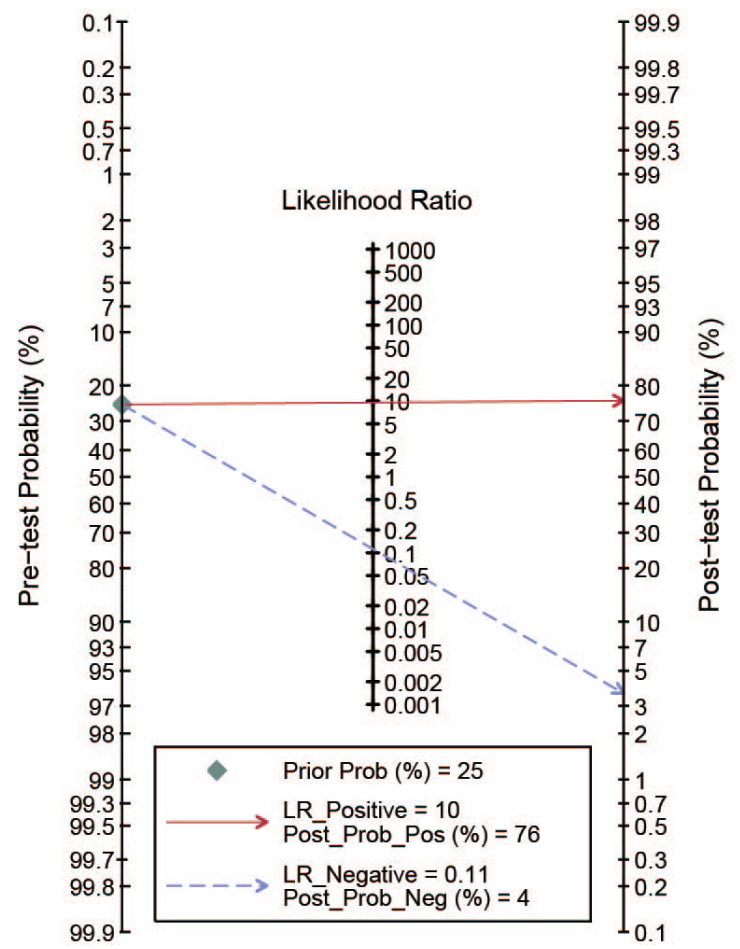

Figure 6. Pre-test probability of microRNA assays in head and neck cancer detection.

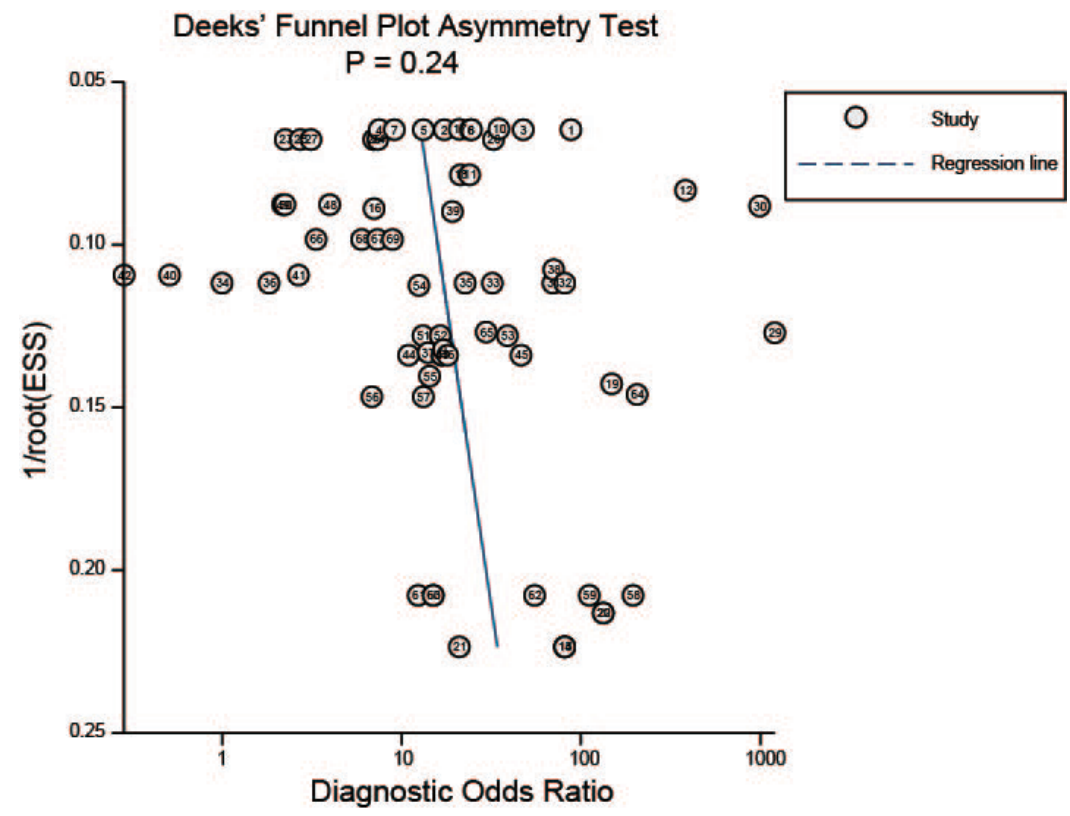

Figure 7. Funnel graph for the assessment of potential publication bias. 
of our knowledge, there is no effective tool to detect early-stage HNC with high accuracy, resulting in late-stage diagnoses. Moreover, regional and distant metastases, which contribute to poor survival rate, may occur during tumor development in esophageal squamous cell carcinoma (ESCC) and thyroid cancer. Therefore, increasing attention has been paid to identify effective biomarkers for the early diagnosis of HNC, and the discovery of miRNAs has opened a new field in cancer detection. Although the literature has confirmed the diagnostic value of miRNA assays, the results are debatable because of the different study designs used. For instance, Zhang et al. (2011) reported that the serum miRNA-31 level in ESCC patients was significantly higher than in healthy controls, and demonstrated its potential as a novel diagnostic marker for the diagnosis of ESCC with $86.7 \%$ sensitivity and $84.3 \%$ specificity. However, for thyroid cancer, miRNA-31 assays yielded $45 \%$ sensitivity and $100 \%$ specificity in the study conducted by Mazeh et al. (2011), which differed greatly from ESCC. When focusing on the diagnostic performance of the miRNA-31 test in oral cancer, Liu et al. (2010) found inconsistent accuracy, with $77 \%$ sensitivity and $80 \%$ specificity through the analysis based on 43 oral cancer patients and 22 healthy individuals. Because the performance of the miRNA assay in HNC detection is still subject to debate, we conducted this meta-analysis to identify the feasibility and the overall diagnostic value of the miRNA test.

In this meta-analysis, the pooled sensitivity was $0.80(95 \% \mathrm{CI}=0.77-0.83)$ and the specificity was $0.80(95 \% \mathrm{CI}=0.76-0.85)$, while the PLR and the NLR were $4.1(95 \% \mathrm{CI}=$ $3.2-5.2)$ and $0.25(95 \% \mathrm{CI}=0.21-0.30)$, respectively, indicating moderate accuracy for the miRNA assays. The DOR, which has an advantage over a single indicator, was $16(95 \% \mathrm{CI}=$ 11-24), while the AUC, which is usually used to summarize overall test performance, was 0.87 $(95 \% \mathrm{CI}=0.84-0.89)$. Overall, these data suggest that miRNAs are promising biomarkers for the diagnosis of $\mathrm{HNC}$ with good accuracy.

There are several obvious advantages of miRNAs when choosing them as candidate clinical biomarkers for the diagnosis of HNC. First, a great deal of effort has gone into applying miRNA assays to HNC patients' diagnosis. Avissar et al. (2009) were the first to demonstrate that miRNA expression showed strong predictive potential in HNC diagnosis, with a high sensitivity of 0.92 and a specificity of 0.93 . Hirajima et al. (2013) measured plasma miRNA-18a levels in 106 esophageal cancer patients and 54 healthy individuals, finding that the miRNA-18a level was significantly higher in patients compared with controls. Gombos et al. (2013) found that certain miRNAs (miRNA-21, -155, -191, R-146a, -221, and -222) were relevant to the diagnosis of oral cancer. Keutgen et al. (2012) investigated the utility of four miRNAs (miRNA-328, -222, R-21, and -197) in indeterminate thyroid fine-needle aspiration lesions, which showed $100 \%$ sensitivity and $86 \%$ specificity for thyroid detection. In the future, more comprehensive research will gain insight into the clinical value of miRNAs.

Second, miRNAs are accessible and stable biomarkers; it is easy to quantify their level of expression and they do not require any invasive procedures. Mitchell et al. (2008) have reported that tumor-derived miRNA exists in a remarkably stable form in human plasma, and miRNAs, which are released in various clinical samples via tumor-derived microvesicles or exosomes, are resistant to RNase activity (Valadi et al., 2007; Skog et al., 2008). Mitchell et al. (2008) also revealed that miRNAs are present in abundance in human serum/plasma/tissue, and can be detected with a simple assay such as qRT-PCR, which makes them well suited to serve as noninvasive biomarkers for tracking diseases.

Third, according to our analysis, miRNA assays are sensitive and specific, especially when using a combination of multiple miRNAs. The subgroup analysis for miRNA profiling 
showed that the PLR for the miRNA test was 10.9 , which meant that the false negative rate was about $9 \%$, while the NLR was 0.11 , which meant that only $11 \%$ of the healthy population was misdiagnosed as cancer patients. Generally, biomarkers with a PLR $>10$ and an NLR $<0.1$ are recognized as excellent indicators of great clinical value; the results of our study indicated that miRNAs could be useful biomarkers. Moreover, Glas et al. (2003) found that DOR ranges from 0 to infinity, so the high DOR value of 99 in our study indicates the significant performance of miRNAs in combination for discriminating HNC patients from non-cancer controls. Notably, the AUC of 0.96 for the miRNA test was also encouraging, because an AUC value of more than 0.97 is associated with excellent accuracy. Thus, miRNA is a promising and noninvasive biomarker, and performs well in both accuracy and discrimination for HNC detection.

Although the results of this meta-analysis were encouraging, some limitations need to be addressed. Firstly, as we know, the major risk factors for HNC are excessive consumption of tobacco and alcohol, but we were not able to carry out a subgroup analysis based on these two variables owing to insufficient data provided by the studies included, which may have had some influence on the outcomes. Secondly, although our data suggest that multiple miRNAs can achieve excellent accuracy and have clinical value, few overlapping types and numbers of miRNAs were found between studies, and the numbers required and the most efficient way to combine the miRNAs have not yet been reported. Hence, further studies investigating such combinations may be needed to improve the miRNA assay outcomes.

Thirdly, owing to the lack of uniform normalization in the methods used in the studies, different results may have arisen from different standardization methods, which certainly would have influenced the results.

In conclusion, although there were limitations, the current evidence suggests that novel and noninvasive miRNAs, especially multiple miRNAs, have potential diagnostic value to serve as screening tools for clinical practice in HNC detection. Larger-scale studies are required to improve the accuracy and explore the most effective combination of miRNAs.

\section{Conflicts of interest}

The authors declare no conflict of interest.

\section{ACKNOWLEDGMENTS}

Research supported by the Science Foundation of Zhejiang Province (\#LY13H060005), the Public Technology Applied Research Projects of Zhejiang Province (\#2014C33254), the General Foundation of Zhejiang Province (\#2013KYA201), the General Research Plan B of Zhejiang Province (\#2012KYB213), and the Shaoxing Science Project (\#2013B70081 and \#2014B70075).

\section{REFERENCES}

Avissar M, Christensen BC, Kelsey KT and Marsit CJ (2009). MicroRNA expression ratio is predictive of head and neck squamous cell carcinoma. Clin. Cancer Res. 15: 2850-2855.

Du T and Zamore PD (2007). Beginning to understand microRNA function. Cell Res. 17: 661-663.

Glas AS, Lijmer JG, Prins MH, Bonsel GJ, et al. (2003). The diagnostic odds ratio: a single indicator of test performance. J. Clin. Epidemiol. 56: 1129-1135. 
Gombos K, Horvath R, Szele E, Juhasz K, et al. (2013). miRNA expression profiles of oral squamous cell carcinomas. Anticancer Res. 33: 1511-1517.

Hardisson D (2003). Molecular pathogenesis of head and neck squamous cell carcinoma. Eur. Arch. Otorhinolaryngol. 260: 502-508.

Hirajima S, Komatsu S, Ichikawa D, Takeshita H, et al. (2013). Clinical impact of circulating miR-18a in plasma of patients with oesophageal squamous cell carcinoma. Br. J. Cancer 108: 1822-1829.

Jemal A, Siegel R, Ward E, Hao Y, et al. (2008). Cancer statistics, 2008. CA Cancer J. Clin. 58: 71-96.

Keutgen XM, Filicori F, Crowley MJ, Wang Y, et al. (2012). A panel of four miRNAs accurately differentiates malignant from benign indeterminate thyroid lesions on fine needle aspiration. Clin. Cancer Res. 18: 2032-2038.

Kitano M, Rahbari R, Patterson EE, Xiong Y, et al. (2011). Expression profiling of difficult-to-diagnose thyroid histologic subtypes shows distinct expression profiles and identify candidate diagnostic microRNAs. Ann. Surg. Oncol. 18: 3443-3452.

Kitano M, Rahbari R, Patterson EE, Steinberg SM, et al. (2012). Evaluation of candidate diagnostic microRNAs in thyroid fine-needle aspiration biopsy samples. Thyroid 22: 285-291.

Kloosterman WP and Plasterk RH (2006). The diverse functions of microRNAs in animal development and disease. Dev. Cell 11: 441-450.

Komatsu S, Ichikawa D, Takeshita H, Tsujiura M, et al. (2011). Circulating microRNAs in plasma of patients with oesophageal squamous cell carcinoma. Br. J. Cancer 105: 104-111.

Liu CJ, Kao SY, Tu HF, Tsai MM, et al. (2010). Increase of microRNA miR-31 level in plasma could be a potential marker of oral cancer. Oral Dis. 16: 360-364.

Liu SS, Wang YS, Sun YF, Miao LX, et al. (2014). Plasma microRNA-320, microRNA-let-7e and microRNA-21 as novel potential biomarkers for the detection of retinoblastoma. Biomed Rep. 2: 424-428.

Liu X, Luo HN, Tian WD, Lu J, et al. (2013). Diagnostic and prognostic value of plasma microRNA deregulation in nasopharyngeal carcinoma. Cancer Biol. Ther. 14.

Lu YC, Chen YJ, Wang HM, Tsai CY, et al. (2012). Oncogenic function and early detection potential of miRNA-10b in oral cancer as identified by microRNA profiling. Cancer Prev. Res. 5: 665-674.

Maclellan SA, Lawson J, Baik J, Guillaud M, et al. (2012). Differential expression of miRNAs in the serum of patients with high-risk oral lesions. Cancer Med. 1: 268-274.

Mazeh H, Mizrahi I, Halle D, Ilyayev N, et al. (2011). Development of a microRNA-based molecular assay for the detection of papillary thyroid carcinoma in aspiration biopsy samples. Thyroid 21: 111-118.

Miska EA (2005). How microRNAs control cell division, differentiation and death. Curr. Opin. Genet. Dev. 15: 563-568.

Mitchell PS, Parkin RK, Kroh EM, Fritz BR, et al. (2008). Circulating microRNAs as stable blood-based markers for cancer detection. Proc. Natl. Acad. Sci. U. S. A. 105: 10513-10518.

Ouyang SB, Wang J, Huang ZK and Liao L (2013). Expression of microRNA-31 and its clinicopathologic significance in oral squamous cell carcinoma. Zhonghua Kou Qiang Yi Xue Za Zhi. 48: 481-484.

Parkin DM (2001). Global cancer statistics in the year 2000. Lancet Oncol. 2: 533-543.

Ries J, Vairaktaris E, Agaimy A, Kintopp R, et al. (2014). miR-186, miR-3651 and miR-494: potential biomarkers for oral squamous cell carcinoma extracted from whole blood. Oncol. Rep. 31: 1429-1436.

Sheinerman KS, Tsivinsky VG and Umansky SR (2013). Analysis of organ-enriched microRNAs in plasma as an approach to development of Universal Screening Test: feasibility study. J. Transl. Med. 11: 304.

Shen R, Liyanarachchi S, Li W, Wakely PE Jr, et al. (2012). MicroRNA signature in thyroid fine needle aspiration cytology applied to "atypia of undetermined significance" cases. Thyroid 22: 9-16.

Skog J, Wurdinger T, van Rijn S, Meijer DH, et al. (2008). Glioblastoma microvesicles transport RNA and proteins that promote tumour growth and provide diagnostic biomarkers. Nat. Cell Biol. 10: 1470-1476.

Stefani G and Slack FJ (2008). Small non-coding RNAs in animal development. Nat. Rev. Mol. Cell Biol. 9: 219-230.

Takeshita N, Hoshino I, Mori M, Akutsu Y, et al. (2013). Serum microRNA expression profile: miR-1246 as a novel diagnostic and prognostic biomarker for oesophageal squamous cell carcinoma. Br. J. Cancer 108: 644-652.

Valadi H, Ekstrom K, Bossios A, Sjostrand M, et al. (2007). Exosome-mediated transfer of mRNAs and microRNAs is a novel mechanism of genetic exchange between cells. Nat. Cell Biol. 9: 654-659.

Vriens MR, Weng J, Suh I, Huynh N, et al. (2012). MicroRNA expression profiling is a potential diagnostic tool for thyroid cancer. Cancer 118: 3426-3432.

Wang Y, Chen M, Tao Z, Hua Q, et al. (2013). Identification of predictive biomarkers for early diagnosis of larynx carcinoma based on microRNA expression data. Cancer Genet. 206: 340-346.

Zeng X, Xiang J, Wu M, Xiong W, et al. (2012). Circulating miR-17, miR-20a, miR-29c, and miR-223 combined as noninvasive biomarkers in nasopharyngeal carcinoma. PLoS One 7: e46367.

Zhang C, Wang C, Chen X, Yang C, et al. (2010). Expression profile of microRNAs in serum: a fingerprint for esophageal 
squamous cell carcinoma. Clin. Chem. 56: 1871-1879.

Zhang T, Wang Q, Zhao D, Cui Y, et al. (2011). The oncogenetic role of microRNA-31 as a potential biomarker in oesophageal squamous cell carcinoma. Clin. Sci. 121: 437-447.

Zhang T, Zhao D, Wang Q, Yu X, et al. (2013). MicroRNA-1322 regulates ECRG2 allele specifically and acts as a potential biomarker in patients with esophageal squamous cell carcinoma. Mol. Carcinog. 52: 581-590.

Zhou SL and Wang LD (2010). Circulating microRNAs: novel biomarkers for esophageal cancer. World J. Gastroenterol. 16: $2348-2354$. 